OPEN ACCESS

Edited by:

Fushun Wang

Nanjing University of Chinese

Medicine, China

Reviewed by:

Xiaolu Zhou,

Shanghai Normal University, China Eleni Vousoura,

American College of Greece, Greece

*Correspondence:

Dexiang Liu

liudexiang@sdu.edu.cn

tThese authors have contributed equally to this work and share first authorship

Specialty section:

This article was submitted to

Psychology for Clinical Settings, a section of the journal Frontiers in Psychology

Received: 15 December 2020 Accepted: 29 March 2021

Published: 29 April 2021

Citation:

Ren Z, Xin Y, Ge J, Zhao Z, Liu D,

Ho RCM and Ho CSH (2021)

Psychological Impact of COVID-19 on

College Students After School Reopening: A Cross-Sectional Study

Based on Machine Learning.

Front. Psychol. 12:641806.

doi: 10.3389/fpsyg.2021.641806

\section{Psychological Impact of COVID-19 on College Students After School Reopening: A Cross-Sectional Study Based on Machine Learning}

\author{
Ziyuan Ren ${ }^{1 \dagger}$, Yaodong Xin ${ }^{2 t}$, Junpeng $\mathrm{Ge}^{3 t}$, Zheng Zhao ${ }^{1}$, Dexiang Liu ${ }^{1 *}$, \\ Roger C. M. Ho ${ }^{4}$ and Cyrus S. H. Ho ${ }^{4}$ \\ ${ }^{1}$ Department of Medical Psychology and Ethics, School of Basic Medicine Sciences, Cheeloo College of Medicine, \\ Shandong University, Jinan, China, ${ }^{2}$ School of Statistics and Management, Shanghai University of Finance and Economics, \\ Shanghai, China, ${ }^{3}$ School of Biology Engineering, Shandong Jianzhu University, Jinan, China, ${ }^{4}$ Department of Psychological \\ Medicine, Yong Loo Lin School of Medicine, National University of Singapore, Singapore, Singapore
}

COVID-19, the most severe public health problem to occur in the past 10 years, has greatly impacted people's mental health. Colleges in China have reopened, and how to prevent college students from suffering secondary damage due to school reopening remains elusive. This cross-sectional study was aimed to evaluate the psychological impact of COVID-19 after school reopening and explore via machine learning the factors that influence anxiety and depression among students. Among the 478 valid online questionnaires collected between September 14th and September 20th, 74 (15.5\%) showed symptoms of anxiety (by the Self-Rating Anxiety Scale), and 155 (32.4\%) showed symptoms of depression (by Patient Health Questionnaire-9). Descriptive analysis of basic personal characteristics indicated that students at a higher grade, having relatives or friends who have been infected, fearing being infected, and having a pessimistic attitude to COVID-19 easily experience anxiety or depression. The Synthetic Minority Oversampling Technique (SMOTE) was utilized to counteract the imbalance of retrieved data. The Akaike Information Criterion (AIC) and multivariate logistic regression were performed to explore significant influence factors. The results indicate that exercise frequency, alcohol use, school reopening, having relatives or friends who have been infected, self-quarantine, quarantine of classmates, taking temperature routinely, wearing masks routinely, sleep quality, retaining holiday, availability of package delivery, take-out availability, lockdown restriction, several areas in school closed due to COVID-19, living conditions in the school, taking the final examinations after school reopening, and the degree to which family economic status is influenced by COVID-19 are the primary influence factors for anxiety or depression. To evaluate the effect of our model, we used 5 -fold cross-validation, and the average area under the curve (AUC) values of the receiver operating characteristic $(\mathrm{ROC})$ curves of anxiety and depression on the test set reached 0.885 and 0.806 , respectively. To conclude, we examined the presence of anxiety and depression symptoms among Chinese college students after school reopening and explored many factors influencing students' mental health, providing reasonable school management suggestions.

Keywords: COVID-19, anxiety, depression, college student, machine learning 


\section{INTRODUCTION}

COVID-19 (coronavirus disease 2019), the most severe public health problem to occur in the past 10 years, has dramatically impacted the medical health service systems worldwide, causing $57,882,183$ confirmed cases and 1,377,395 confirmed deaths up to 22 November 2020 (World Health Organization, 2020). It was first discovered in Wuhan, the Hubei province's capital city, China, and rapidly spread to other regions (Guan et al., 2020a,b). Indeed, after strict regulations were administrated across China, including quarantine, mask-wearing, large-scale nucleic acid assay, etc., the situation of COVID-19 in China significantly improved (Tang et al., 2020; Tu et al., 2020). From 19 November to 21 November 2020, the number of new cases discovered in China was 11 (National Health Commission of the People's Republic of China, 2020). However, more psychologists find that psychological problems, especially posttraumatic anxiety and depression, also matter after this dreadful disaster (Mazza et al., 2020; Pappa et al., 2020; Shader, 2020; Vindegaard and Benros, 2020).

Due to the severity of COVID-19 in the first half of this year, universities across China were closed, and all the students stayed at home and took online courses. In May and June, several universities in the so-called low-risk area reopened. Their students came back to attend their final exams, while most universities did not reopen until September 2020 (Ministry of Education of the People's Republic of China, 2020; People's Daily, 2020). Additionally, strict regulations were announced to protect college students from being infected (Ministry of Education of the People's Republic of China, 2020). For example, students must wear masks and accept temperature testing before they can come into the classroom. Besides, delivery services and free entry to the campus are no longer allowed.

Even before the COVID-19, college students are susceptible to mental health challenges facing unprecedented levels of distress, and early adulthood is one of the peak periods for many mental disorders (de Girolamo et al., 2012). Seventy-five percent of patients with mental health disorders had the first onset before 25 (Kessler et al., 2007). In China, it is reported that the prevalence of suicidal ideation was $9.2 \%$ among college students in Jilin province, 2019, and the two most significant risk factors were being a senior and family relationship (Wang et al., 2019). College students play a crucial role in the development of a country. Therefore, with media attention on the college campus, the high rates of mental disorder prevalence have become a growing public health problem in many countries. During the COVID-19, young adults and college students faced more mental challenges, including academic pressure, employment pressure, and family pressure. Many previous studies demonstrated that COVID-19 has led to profound mental and behavioral changes among college students (Alemany-Arrebola et al., 2020; Huckins et al., 2020; Ma et al., 2020; Zhai and Du, 2020). Cao et al. performed a cross-sectional study in China and found that $0.9 \%$ of the respondents were experiencing severe anxiety, $2.7 \%$ were experiencing moderate anxiety, and $21.3 \%$ were experiencing mild anxiety (Cao et al., 2020). Similarly, college students' anxiety and depression rates during the early stages of COVID-19 were not optimistic in the United States (Kecojevic et al., 2020) and
Bangladesh (Khan et al., 2020). It is necessary and urgent to improve college students' mental status, and any exacerbation due to strict school regulations after school reopening is unacceptable (Giannopoulou et al., 2020; Kalok et al., 2020).

Whether universities should implement strict regulations remains controversial (Beijing News, 2020). Some think it is irrational to sacrifice students' mental health to prevent COVID19 transmission. Others believe the strict regulation will not cause mental damage to college students. COVID-19 will not be the last pandemic, so it is of great significance to accumulate experience to avoid severe damage to college students' mental health during the next pandemic event. However, no published articles have investigated the current psychological status of students under these regulations. To this end, we conducted this cross-sectional study via an online questionnaire to ascertain the psychological impact after school reopening on students among five universities across China. Further, we performed machine learning to screen out risk and protective factors that influence the college students' mental health status, including school regulation, family situation, and personal living styles. These influence factors may provide some sensible advice for the school administrative department to prevent college students from mental diseases.

\section{MATERIALS AND METHODS}

\section{Ethics Statement}

This cross-sectional study was approved by the Ethics Committee of Shandong University as a human-involved study with the serial number ECSBMSSDU2020-1-056. The Declaration of Helsinki was strictly followed, and no identifying information was collected. This cross-sectional study's data collection was conducted between September 14th and September 20th, mainly among five universities across China: Shandong University, Shandong Normal University, Qingdao University (Qingdao, Shandong province), Shanghai Tech University, and Shanghai University of Finance and Economics (Shanghai). Because of COVID-19, an online anonymous questionnaire website (www.wenjuan.com) was used. Therefore, no formal consent was acquired. All participants voluntarily ticked off the informed consent in the first item.

\section{Participants}

Among the 548 invited subjects, 508 subjects were invited to complete the questionnaire by the investigators, and 40 were invited by a free open access online questionnaire distribution platform, www.wjx.cn. Among all the 548 retrieved questionnaires, 478 were valid to study further. Two retrieved questionnaires left blanks, 66 left obvious and invalid options, and 2 had an IP address outside China.

\section{Designed Questionnaire Basic Personal Characteristics}

Two sets of basic personal characteristics were listed in the questionnaire: demographic characteristics and personal perspectives on COVID-19. Demographic characteristics included gender, major, grade, and family location. Personal perspectives on COVID-19 included fear of being infected, 
attitude to COVID-19, history of psychological counseling, and need for psychological counseling. In this study, "psychological counseling" means college students received psychological counseling from their school. In China, the impact of the COVID-19 on college students' mental health has drawn public attention. After the school reopening, the university might provide psychological counseling for all students. The detailed options for each question are presented in Table 1.

\section{Assessment of Anxiety and Depression}

The classic Zung's Self-rating Anxiety Scale (SAS) was used to evaluate the participants' anxiety degree (Zung, 1971); it includes 20 self-reported items about moods, sleep, sense of pain, etc. After the standardized scoring algorithm, four anxiety degree grades were defined. A score of 20-49 was considered as no anxiety, 50-59 as mild anxiety, 60-69 as moderate anxiety, and $70-80$ as severe anxiety. Zhou et al. demonstrated that the reliability and validity of SAS applied in Chinese college students were acceptable. The criterion defining normal/mild/moderate/severe anxiety was suitable for Chinese (Yongan, 2012). The Cronbach's alpha was 0.906 for SAS in the current study.

The Patient Health Questionnaire-9 (PHQ-9) was used to evaluate the depression degree (Kroenke et al., 2001); it includes nine self-reported items with a score of $0-4$ for each question. After the standardized scoring algorithm, five depression degree grades were defined. A score of $0-4$ was considered as no depression, 5-9 as mild depression, 10-14 as moderate depression, 15-19 as moderate to severe depression, and $20-27$ as severe depression. This scale has been confirmed to be a reliable and valid tool in assessing mental health in Chinese adolescents (Xingchen et al., 2014). In this study, the Cronbach's alpha for PHQ-9 was 0.918.

\section{Influence Factor Selection}

All the influence factors included in our questionnaire were acquired from a 20 -subject pre-survey. We randomly invited 20 subjects included in the pre-survey from Shandong University. Two investigators (Y.X and J.P) searched the Pubmed and retrieved 30 potential influence factors. We asked 20 subjects whether they agreed a certain factor might significantly influence students' psychological condition. Therefore, we selected those factors which are agreed by most subjects (>10/20). After considering all the retrieved influence factors comprehensively, three sets of questions were included in our questionnaire: school regulations, family situation, and personal living style. School regulations mean the regulations announced during COVID-19 to prevent disease transmission across schools, including school reopening, routine mask-wearing, routine temperature-taking, several areas in school being closed due to COVID-19, final examinations being taken after school reopening, retaining holiday (whether to shorten or cancel the holiday after school reopening), availability of package delivery (after school reopening), take-out food availability (after school reopening), lockdown restriction (whether to allow free access to campus after school reopening), quarantine of classmates (after school reopening), and self-quarantine after school reopening. The "retaining holiday (whether to shorten or cancel the holiday after school reopening)" here refers to the fact that a number of universities in China have shortened or canceled some holidays in order to minimize the students' total in-school time after school reopening during COVID-19. "Holiday" here includes weekends and statutory holidays in China. The family situation means the impact of COVID-19 on family, including having relatives or friends who have been infected and the degree to which family economic status was influenced by COVID-19. The personal living style includes exercise frequency, alcohol use, sleep quality, and satisfaction with school living conditions. The detailed options for each question are presented in Table 2.

\section{Statistics}

Correlation analysis was performed for univariate analysis to primarily determine whether factors have relations with students' anxiety or depression conditions in a descriptive view. More specifically, for independent variables from two categories or multiple unordered categories, we used the chi-square test. For the cells in which the samples numbered $<5$, we used Fisher's exact test. For explanatory variables from multiple ordered categories, we used Somer's $d$ to measure the consistency between the two (that is, whether the two tend to move in the same or opposite directions).

Logistic regression was performed for multivariate analysis to determine the association between a particular factor and students' psychological status quantitively, other factors being equal. First, we divided the samples into five equal parts in order to perform 5-fold cross-validation. Second, the Synthetic Minority Oversampling Technique (SMOTE) was performed on the training dataset to preprocess the retrieved data. As is acknowledged to all, the number of positive and negative samples of medical data is often uneven, which could strongly affect the effectiveness of the Logistic regression model. SMOTE is an oversampling algorithm that generates extra samples based on the original dataset. By setting a specific scale, SMOTE can make the dataset balanced using methods of oversampling. The Akaike Information Criterion (AIC) and binary logistic regression were performed to explore significant influence factors. The accuracy, sensitivity (recall), specificity $($ accuracy $=(\mathrm{TP}+\mathrm{TN}) /(\mathrm{TP}+$ $\mathrm{TN}+\mathrm{FP}+\mathrm{FN})$, sensitivity $=\mathrm{TP} /(\mathrm{TP}+\mathrm{FN})$, specificity $=\mathrm{TN} /(\mathrm{TN}+\mathrm{FP})$, where $\mathrm{TP}$ is correct positive assignments, $\mathrm{TN}$ is correct negative assignments, FP is incorrect positive assignments, and FN is incorrect negative assignments) and area under the curve (AUC) values of the receiver operating characteristic (ROC) curves were used to evaluate the machine learning model.

Data collection and descriptive analysis were performed using Excel 2016 (Microsoft, Washington, D.C., US). Univariate analyses were performed using IBM SPSS Statistics 24.0 (International Business Machines Corporation, Armonk, NY, USA). Multivariate analyses were performed using the $\mathrm{R}$ language(R Core Team, 2020). A $P$-value $<0.05$ was considered statistically significant. 
TABLE 1 | Descriptive analysis of basic characteristics.

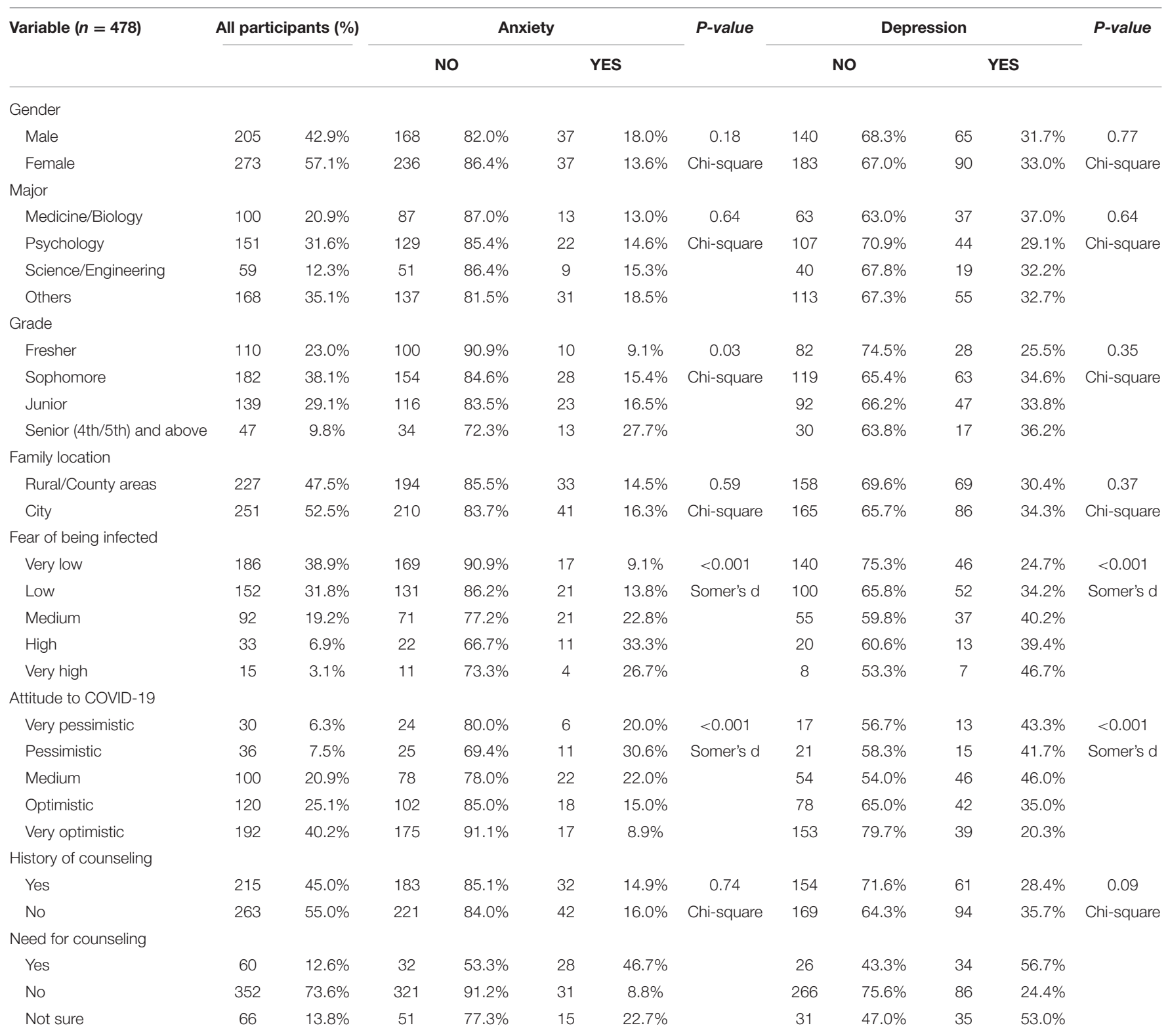

Counseling here means psychological counseling received from school or from others. Somer's $d$ was used to measure the consistency of ordered categories. Chi-square test was used to measure correlations between unordered categories.

\section{RESULTS}

\section{Presence of Anxiety and Depression Symptoms}

This survey's response variables were anxiety and depression evaluated by SAS and PHQ-9, respectively. Among all the 478 valid subjects, $74(15.5 \%)$ showed symptoms of anxiety (among which $4(0.8 \%)$ showed severe anxiety, 15 (3.1\%) showed moderate anxiety, and 55 (11.5\%) showed mild anxiety). Besides, 155 (32.4\%) showed symptoms of depression (among which 9 (1.9\%) showed severe depression, 26 (5.4\%) showed moderate to severe depression, 46 (9.6\%) showed moderate depression, and
74 (15.5\%) showed mild depression). We divided subjects into two sets-anxiety (depression) and normal (normal) - for further influence factor exploration.

\section{Descriptive Analysis of Basic Personal Characteristics}

The basic personal characteristics of the 478 valid subjects are displayed in Table 1. College students at a higher grade, fear being infected, and have a more pessimistic attitude to COVID19 were more likely to report anxiety or depression, while gender, major, and family location did not significantly differ. After comparing 215 (45\%) subjects who have received psychological 
TABLE 2 | Descriptive statistics of influence factors.

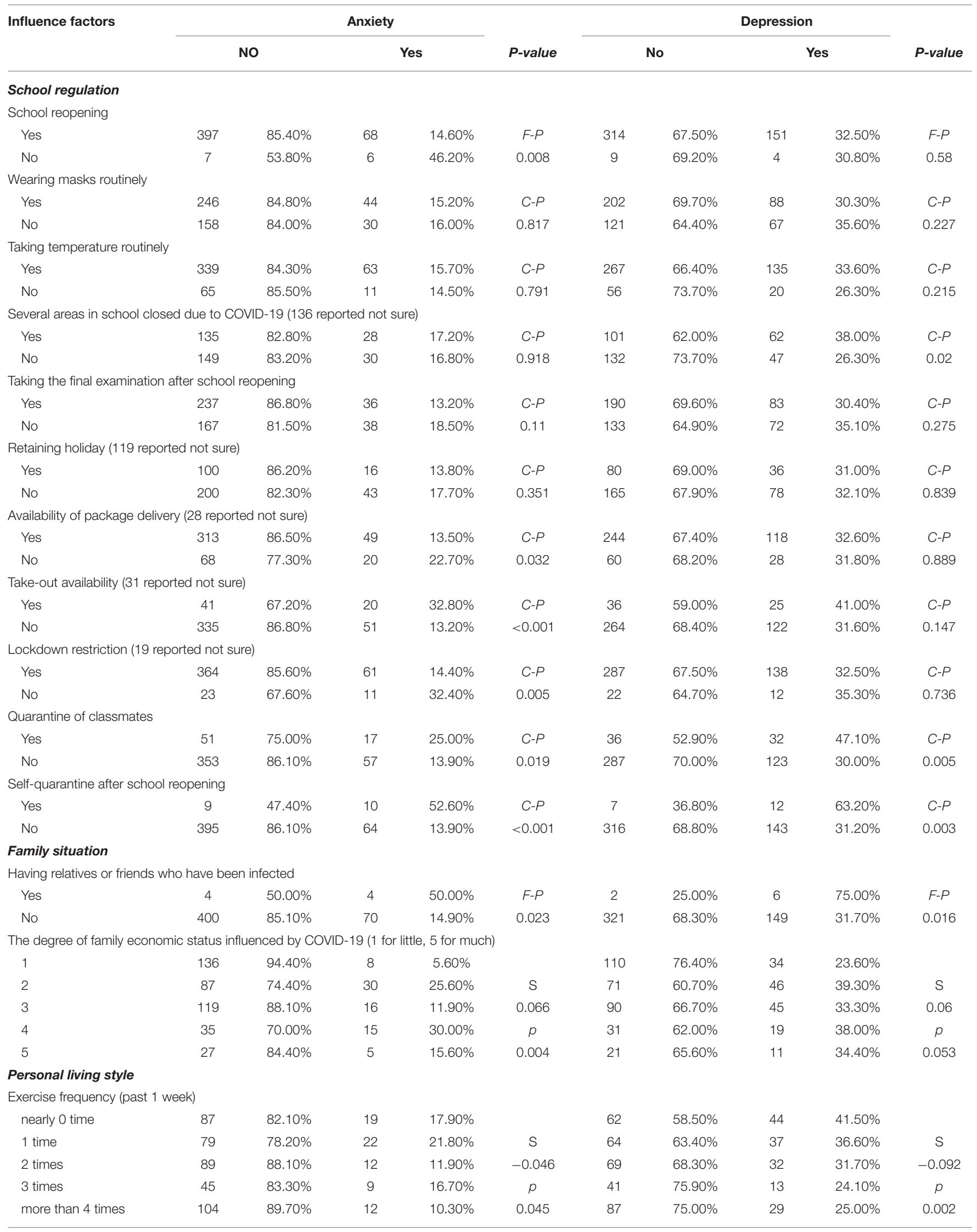


TABLE 2 | Continued

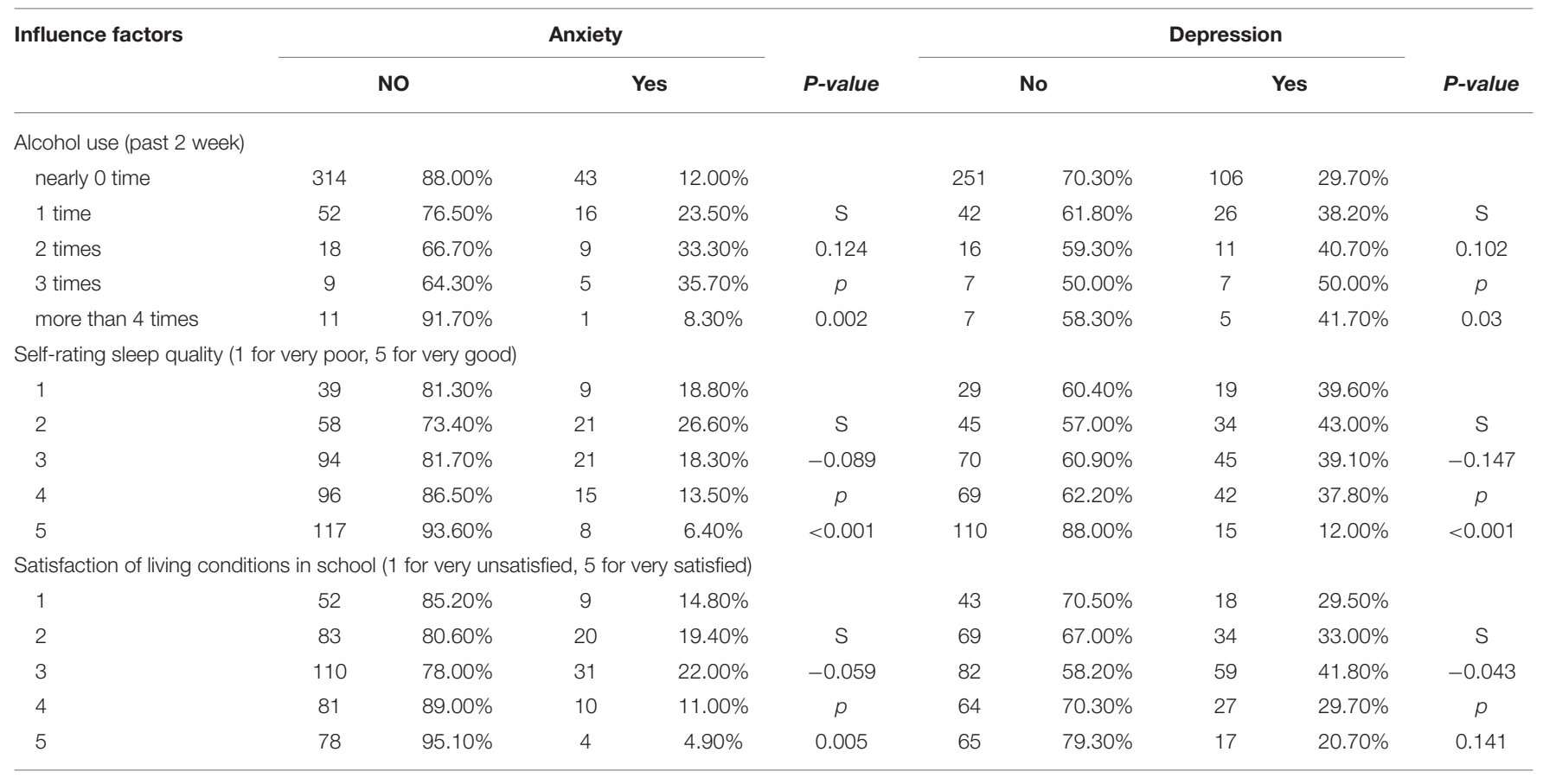

F-P, P-value of Fisher's test; C-P, P-value of the Chi-square test; S, Somer's d; $p$, P-value of Somer's d.

counseling with the other students who did not, no significant difference in the presence of expression/anxiety symptoms was observed for students who had received counseling ( $p=0.74$ for anxiety, and $p=0.09$ for depression) compared with the control group, which means that the effect of current psychological counseling is limited. Besides, 60 (12.6\%) students reported that they need psychological counseling. Notably, 66 (13.8\%) students reported that they had no idea whether they need professional psychological counseling; the presence of anxiety was $22.7 \%$, and that of depression was $53 \%$ in this group, which is nearly three times the presence of anxiety and two times the presence of depression observed in the group of students who reported that they did not need counseling. Therefore, the group of students who are not sure whether they need psychological counseling appears particularly vulnerable for experiencing clinically significant depression or anxiety.

\section{Descriptive Analysis of Influence Factors}

The characteristics of the 17 influence factors in our questionnaires are displayed in Table 2. In the set of school regulations, school reopening, several areas in schools being closed due to COVID-19, lockdown restriction, and availability of package delivery alleviated college students' anxiety or depression. A significant relationship between depression or anxiety and take-out availability, quarantine of classmates, and self-quarantine after school reopening was found $(p<0.05)$. Other risk factors were of no statistical significance $(p>0.05)$. In terms of family situation, having relatives or friends who have been infected and the family economic status being influenced to a strong degree by COVID-19 were significantly related to psychological problems. Finally, in personal living style, students who exercise more, drink less, sleep better, and are satisfied with their living conditions reported a healthier psychological state.

\section{Construction of the Logistic Regression Model}

SMOTE was performed to counteract the imbalance of the retrieved data (15.5\% of subjects showed a symptom of anxiety, and $32.4 \%$ of subjects show a symptom of depression). When using SMOTE, we need to determine three parameters: $k$, perc.over, and perc.under. $k$ represents the number of nearest neighbors used to generate new instances of the minority classes. perc.over decides how many additional cases to generate from the minority classes (known as oversampling). perc.under decides how many extra cases from the majority classes are selected for each case generated from the minority class (known as undersampling). As shown in Figure 1A, for the SAS dataset, when $\underline{k}=$ 6 , perc.over $=500$, and perc.under $=120$, the AUC reaches the maximum. Similarly, for the PHQ9 dataset, when $k=2$, perc.over $=400$, and perc.under $=125$, the AUC reaches the maximum (Figure 1B). Since we had divided the dataset into five equal parts, we selected one part as the test set each time and using SMOTE to process the other four groups. A new dataset was thus utilized in the construction of the logistic regression model for anxiety and depression.

The AIC (Table 3) was applied to select proper influence factors for inclusion in the logistic regression model. Among the 17 influence factors acquired from a 20 -subject pre-survey, 13 factors (taking temperature routinely, retaining holiday, selfrated sleep quality, taking the final examination after school 

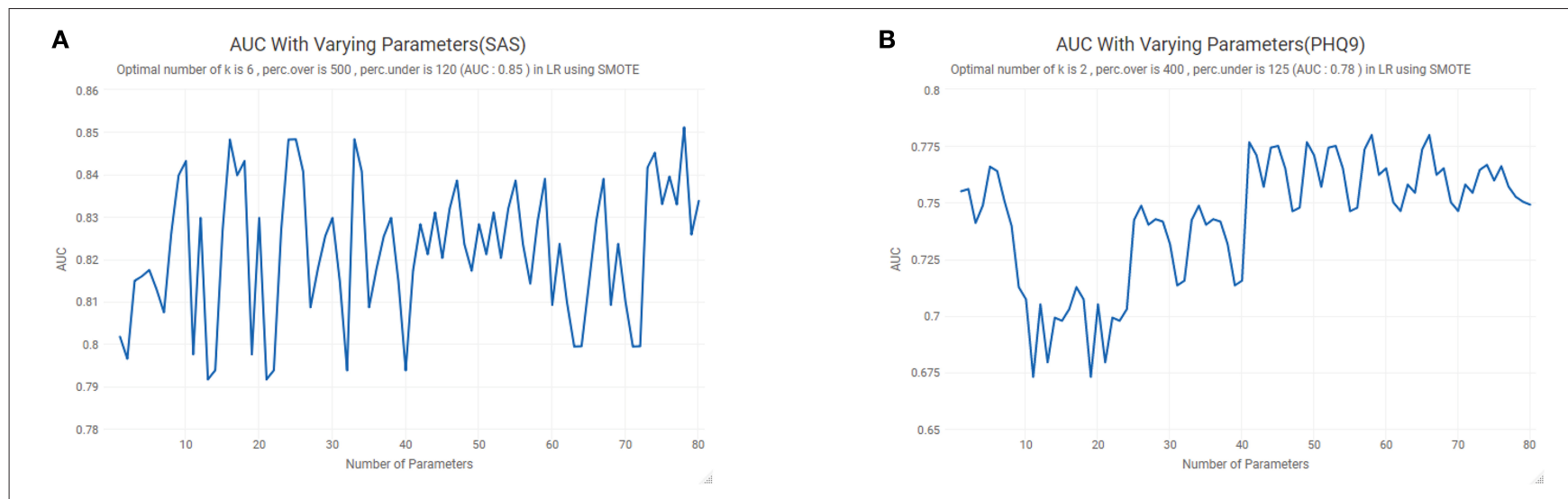

FIGURE 1 | The Synthetic Minority Oversampling Technique (SMOTE): (A) anxiety; (B) depression. AUC, area under the curve.

reopening, lockdown restriction, exercise frequency, quarantine of classmates, take-out availability, alcohol use, availability of package delivery, school reopening, self-quarantine after school reopening, and the degree to which family economic status is influenced by COVID-19) were extracted according to the AIC for anxiety, while 15 factors (routinely wearing masks, having relatives or friends who have been infected, satisfaction with living conditions in the school, taking temperature routinely, several areas in school being closed due to COVID-19, selfrated sleep quality, taking the final examination after school reopening, lockdown restriction, exercise frequency, quarantine of classmates, take-out availability, alcohol use, availability of package delivery, self-quarantine after school reopening, and the degree to which family economic status is influenced by COVID-19) were extracted for depression.

We used a conventional generalized linear model, Logistic regression, to analyze the data after selecting factors. For each factor, we set the first level as the control group. The results of the logistic regression are presented in Table 4. The binary logistic regression finally extracted 12 significant influence factors (excluding sleep quality from the list of 13 above) for anxiety and 12 (excluding taking temperature routinely, taking the final examination after school reopening, and availability of package delivery from the list of 15 above) for depression $(p<0.05)$.

\section{Evaluation of the Logistic Regression Model}

When applying a logistic regression model, it is crucial to avoid overfitting. To evaluate our model effectively, we first divided the dataset into five equal parts, using four of them to train a regression model. Then, we tested the generated model to see whether there was a significant difference. The ROC curves of 5-fold cross-validation were plotted to evaluate our logistic regression model (Figure 2), where the shadow area represents the $95 \%$ confidence interval of the ROC curve. The anxiety model's average accuracy was $81.42 \%$, and that of the depression model was $73.5 \%$. On the test dataset, the average AUC of the anxiety model was 0.885 , and that of the depression model was
0.806 , which indicates that the predictive power of our models is excellent. The average sensitivity (recall) of the models reached 83.21 and $75.3 \%$, respectively. The average specificity of the models reached 80.38 and $71.80 \%$, respectively. The sensitivity and specificity were both acceptable.

\section{DISCUSSION}

In this study, we performed a cross-sectional survey to investigate the presence of anxiety and depression symptoms among Chinese college students after school reopening and explored a series of factors influencing students' mental health.

First, the descriptive statistics of basic personal characteristics were utilized to test whether these basic characteristics would affect students' emotional status. The result shows that students having relatives or friends who have been infected, fearing being infected, having a more pessimistic attitude toward COVID-19 were more likely to report psychological problems. Moreover, we found that students at a higher grade easily got anxious and depressed. First, senior students must participate in a practicum, which has been demonstrated as a risk factor for stress and anxiety (Cheung et al., 2016). Besides, senior students faced more mental challenges, including greater academic pressure, graduation pressure, etc. Furthermore, due to the pandemic of COVID-19, these pressures were amplified. Of the participants, $60(12.6 \%)$ reported needing psychological help, and 66 (13.8\%) reported that they had no idea whether they needed psychological help. These students would be more vulnerable than others for lacking awareness of the importance of psychological health and not getting prompt treatment. Therefore, it is necessary to give students universal mental health education. Besides, among the 478 subjects, 215 (45\%) had received psychological counseling from school. However, it had not resulted in a significant improvement of their mental health, which indicates that the effect of current psychological help for college students is limited. Several potential reasons probably cause this. Firstly, the effect of current psychological help, especially online counseling, for college students is limited. Many Chinese university counselors 
TABLE 3 | The Akaike Information Criterion (AIC) values of the 17 influence factors for anxiety and depression.

\begin{tabular}{|c|c|c|c|c|}
\hline In/Out & Influence factor & Df & Deviance & AIC \\
\hline \multicolumn{5}{|l|}{ Anxiety } \\
\hline & $<$ none $>$ & & 447.52 & 507.52 \\
\hline Out & wearing masks routinely & 1 & 446.65 & 508.65 \\
\hline Out & $\begin{array}{l}\text { having relatives or friends who have } \\
\text { been infected }\end{array}$ & 1 & 447.49 & 509.49 \\
\hline Out & $\begin{array}{l}\text { satisfaction of living conditions in } \\
\text { school ( } 1 \text { for very unsatisfied, } 5 \text { for } \\
\text { very satisfied) }\end{array}$ & 4 & 441.6 & 509.6 \\
\hline $\ln$ & taking temperature routinely & 1 & 451.68 & 509.68 \\
\hline Out & $\begin{array}{l}\text { several areas in school closed due } \\
\text { to COVID-19 }\end{array}$ & 1 & 446.72 & 510.72 \\
\hline $\ln$ & retaining holiday & 1 & 456.94 & 512.94 \\
\hline In & $\begin{array}{l}\text { self-rating sleep quality ( } 1 \text { for very } \\
\text { poor, } 5 \text { for very good) }\end{array}$ & 4 & 463.64 & 515.64 \\
\hline $\ln$ & $\begin{array}{l}\text { taking final examination after school } \\
\text { reopening }\end{array}$ & 1 & 457.95 & 515.95 \\
\hline In & lockdown restriction & 1 & 462.73 & 518.73 \\
\hline $\ln$ & exercise frequency (past 1 week) & 4 & 472.48 & 524.48 \\
\hline $\ln$ & quarantine of classmates & 1 & 467.86 & 525.86 \\
\hline In & take-out food availability & 1 & 470.69 & 526.69 \\
\hline $\ln$ & alcohol use (past 2 week) & 4 & 476.08 & 528.08 \\
\hline $\ln$ & availability of package delivery & 1 & 472.56 & 528.56 \\
\hline $\ln$ & school reopening & 1 & 473.96 & 531.96 \\
\hline In & $\begin{array}{l}\text { self-quarantine after school } \\
\text { reopening }\end{array}$ & 1 & 476.11 & 534.11 \\
\hline In & $\begin{array}{l}\text { the degree of family economic } \\
\text { status influenced by COVID-19 ( } 1 \\
\text { for little, } 5 \text { for much) }\end{array}$ & 4 & 482.45 & 534.45 \\
\hline \multicolumn{5}{|c|}{ Depression } \\
\hline & $<$ none $>$ & & 1205.8 & 1275.8 \\
\hline $\ln$ & $\begin{array}{l}\text { taking the final examination after } \\
\text { school reopening }\end{array}$ & 1 & 1208.5 & 1276.5 \\
\hline Out & retaining holiday & 1 & 1203 & 1277 \\
\hline $\ln$ & taking temperature routinely & 1 & 1209.2 & 1277.2 \\
\hline Out & school reopening & 1 & 1205.8 & 1277.8 \\
\hline In & $\begin{array}{l}\text { several areas in school closed due } \\
\text { to COVID-19 }\end{array}$ & 1 & 1212.5 & 1278.5 \\
\hline $\ln$ & availability of package delivery & 1 & 1213.7 & 1279.7 \\
\hline $\ln$ & exercise frequency (past 1 week) & 4 & 1227 & 1289 \\
\hline $\ln$ & $\begin{array}{l}\text { the degree of family economic } \\
\text { status influenced by COVID-19 (1 } \\
\text { for little, } 5 \text { for much) }\end{array}$ & 4 & 1230.2 & 1292.2 \\
\hline $\ln$ & take-out food availability & 1 & 1228.2 & 1294.2 \\
\hline In & $\begin{array}{l}\text { satisfaction of living conditions in } \\
\text { school ( } 1 \text { for very unsatisfied, } 5 \text { for } \\
\text { very satisfied) }\end{array}$ & 4 & 1233.9 & 1295.9 \\
\hline In & alcohol use (past 2 week) & 4 & 1240.1 & 1302.1 \\
\hline $\ln$ & wearing masks routinely & 1 & 1235.3 & 1303.3 \\
\hline $\ln$ & lockdown restriction & 1 & 1237.3 & 1303.3 \\
\hline In & $\begin{array}{l}\text { having relatives or friends who have } \\
\text { been infected }\end{array}$ & 1 & 1238.5 & 1306.5 \\
\hline In & quarantine of classmates & 1 & 1239 & 1307 \\
\hline $\ln$ & $\begin{array}{l}\text { self-quarantine after school } \\
\text { reopening }\end{array}$ & 1 & 1256.2 & 1324.2 \\
\hline In & $\begin{array}{l}\text { self-rating sleep quality ( } 1 \text { for very } \\
\text { poor, } 5 \text { for very good) }\end{array}$ & 4 & 1272.8 & 1334.8 \\
\hline
\end{tabular}

"In" means included in the logistic regression model; "Out" means excluded from the logistic regression model; DF, degrees of freedom. would need training in psychological service. Moreover, it may still require a longer time to observe therapeutic changes of the psychological survey. Universities are essential in dealing with the mental status of college students (Zhai and Du, 2020). It is impractical to provide face-to-face professional psychological counseling to every college student due to financial limitations and psychologists' numbers. Besides selecting students in need by way of the influence factors discussed above, tele-counseling is particularly important in this area. Previous studies have reported that tele-counseling or digital mental health interventions have developmental prospects (Levin et al., 2016, 2017; Lattie et al., 2019). However, some also reported that the current situation of college psychological centers' website effectiveness is compromised. Seidel et al. reported that only half of all 138 analyzed websites provided information about remote counseling. Approximately two-thirds of them had directions for students experiencing a mental health emergency (Seidel et al., 2020). Indeed, how to provide professional psychological help to students in need remains controversial (Lungu and Sun, 2016; Webermann and Murphy, 2020).

Second, univariate and multivariate analysis extracted 17 significant factors influencing college students' mental status. Among these influence factors, four factors of healthier personal lifestyles-higher exercise frequency, lower alcohol use, higher sleep quality, and higher satisfaction with living conditions in the school-were closely related to a lower risk of psychological problems. Several studies in the literature have demonstrated that these influence factors play a crucial role in public mental health (Walsh, 2011; Velten et al., 2018; Oftedal et al., 2019). Notably, it was reported that sleep problems among adolescents and young adults during the COVID-19 epidemic, especially college students, are common and negatively associated with students' projections of trends in COVID-19 (Zhou et al., 2020). Zhang et al. found that sleep problems may mediate the pandemic's impact on mental health (Zhang et al., 2020). Therefore, more attention should be paid to insomnia currently. As for the two family situation influence factors, having relatives or friends who have been infected and unstable family income would cause psychological problems. In terms of the 11 school regulation influence factors, quarantine was a robust factor associated with clinical symptoms of anxiety and depression (Khan et al., 2020; Tang et al., 2020,a; Xin et al., 2020) compared with the control group, suggesting that reducing unnecessary quarantine measures can effectively improve students' mental health. The protective regulations, such as lockdown restriction, were mostly related to better mental health, unexpected before this investigation. This result suggests that college students would rather endure some inconvenience in daily life than be probably infected with the virus, except for some daily necessities, such as delivery and retaining holiday. With the rate of the virus spread slowing down, schools at all levels are reopening. Although the pandemic situation has been much improved, finding the balance between protecting students from coronavirus infection and preventing students from the pressure of delayed schooling, compromised living conditions, and physical health is challenging for policymakers. In this study, we screened the significant influence factors associated with 
TABLE 4 | Binary logistic regression model.

\begin{tabular}{|c|c|c|c|c|}
\hline & \multicolumn{2}{|c|}{ Anxiety } & \multicolumn{2}{|c|}{ Depression } \\
\hline \multicolumn{5}{|l|}{ School reopening } \\
\hline Yes & reference & & none & \\
\hline No & $21.99(6.84,70.68)$ & $<0.001$ & & \\
\hline Yes & & & reference & \\
\hline No & & & $2.33(1.71,3.17)$ & $<0.001$ \\
\hline \multicolumn{5}{|c|}{ Taking temperature routinely } \\
\hline Yes & reference & & reference & \\
\hline No & $1.88(1.10,3.23)$ & 0.021 & $0.7(0.47,1.03)$ & 0.067 (out) \\
\hline Yes & reference & & reference & \\
\hline No & $2.40(1.53,3.79)$ & $<0.001$ & $1.27(0.96,1.7)$ & 0.099 (out) \\
\hline \multicolumn{5}{|l|}{ Retaining holiday } \\
\hline Yes & reference & & none & \\
\hline No & $1.85(1.07,3.21)$ & 0.028 & & \\
\hline \multicolumn{5}{|c|}{ Availability of package delivery } \\
\hline Yes & reference & & reference & \\
\hline No & $3.53(2.06,6.03)$ & $<0.001$ & $1.13(0.77,1.64)$ & 0.5329 (out) \\
\hline \multicolumn{5}{|c|}{ Tale-out food availability } \\
\hline Yes & reference & & reference & \\
\hline \multicolumn{5}{|c|}{ Self-quarantine after school reopening } \\
\hline Yes & reference & & reference & \\
\hline No & $0.15(0.07,0.33)$ & $<0.001$ & $0.13(0.07,0.26)$ & $<0.001$ \\
\hline \multicolumn{5}{|l|}{ Family situation } \\
\hline \multicolumn{5}{|c|}{ Having relatives or friends who have been infected } \\
\hline Yes & none & & reference & \\
\hline No & & & $0.08(0.03,0.24)$ & $<0.001$ \\
\hline \multicolumn{5}{|c|}{ The degree of family economic status influenced by COVID-19 ( 1 for little, 5 for much) } \\
\hline 1 & reference & & reference & \\
\hline 2 & $4.56(2.31,9.00)$ & $<0.001$ & $1.86(1.23,2.8)$ & 0.003 \\
\hline 3 & $1.00(0.5,2.04)$ & 0.990 & $1.37(0.92,2.05)$ & 0.124 \\
\hline 4 & $4.7(2.15,10.28)$ & $<0.001$ & $3.28(1.91,5.64)$ & $<0.001$ \\
\hline 5 & $4.35(1.69,11.18)$ & 0.002 & $2.26(1.26,4.06)$ & 0.006 \\
\hline \multicolumn{5}{|l|}{ Personal living style } \\
\hline \multicolumn{5}{|c|}{ Exercise frequency (past 1 week) } \\
\hline nearly 0 time & reference & & reference & \\
\hline 1 time & $1.48(0.80,2.73)$ & 0.215 & $0.65(0.43,1.01)$ & 0.053 \\
\hline 2 times & $0.54(0.28,1.03)$ & 0.060 & $0.75(0.49,1.15)$ & 0.185 \\
\hline 3 times & $0.90(0.40,2.03)$ & 0.802 & $0.45(0.26,0.78)$ & 0.004 \\
\hline more than 4 times & $0.37(0.18,0.74)$ & 0.005 & $0.42(0.28,0.63)$ & $<0.001$ \\
\hline
\end{tabular}




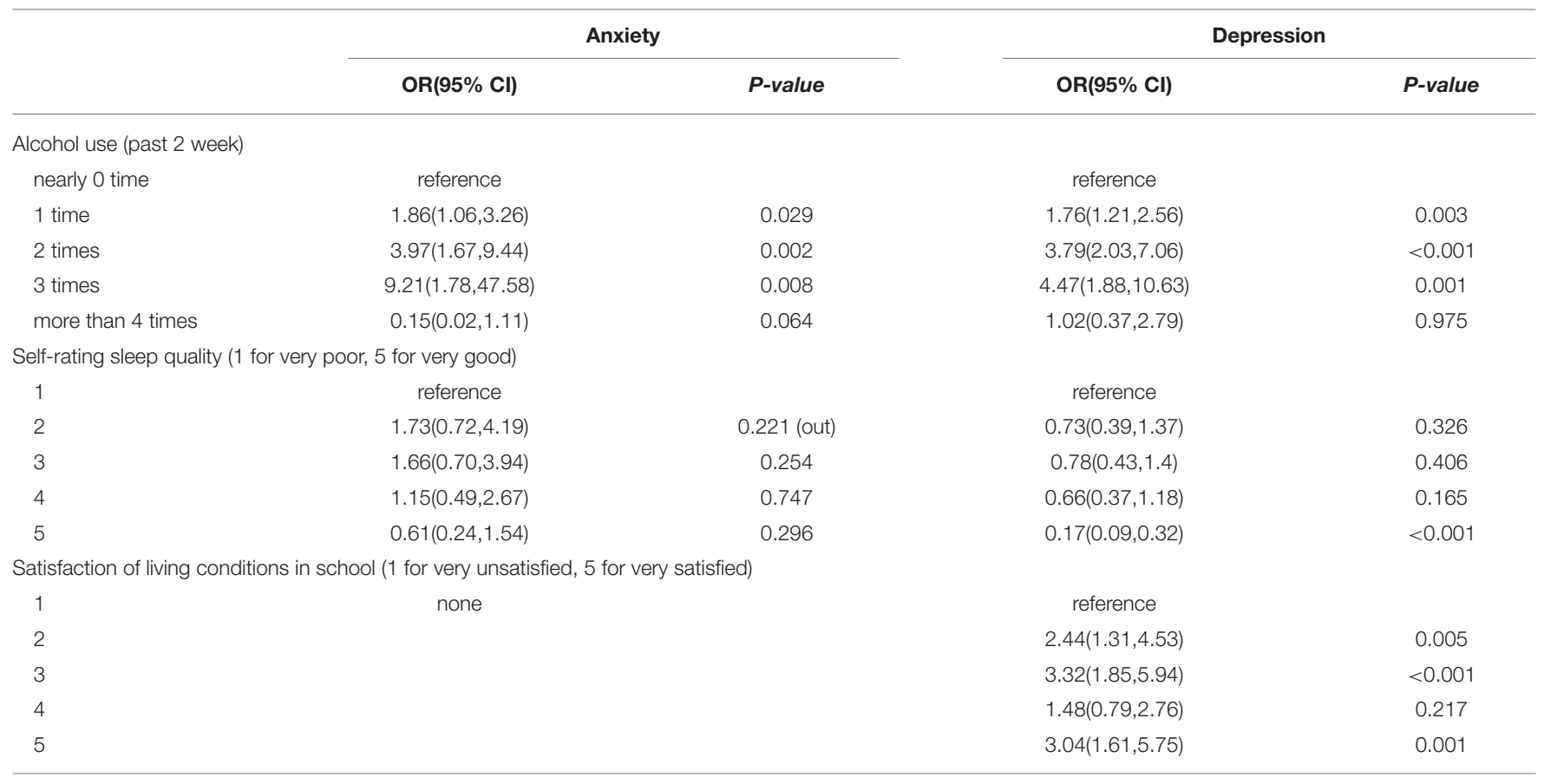

OR, odds ratio; Cl, confidence interval; "out" means no statistical significance; "none" means excluded by AlC.
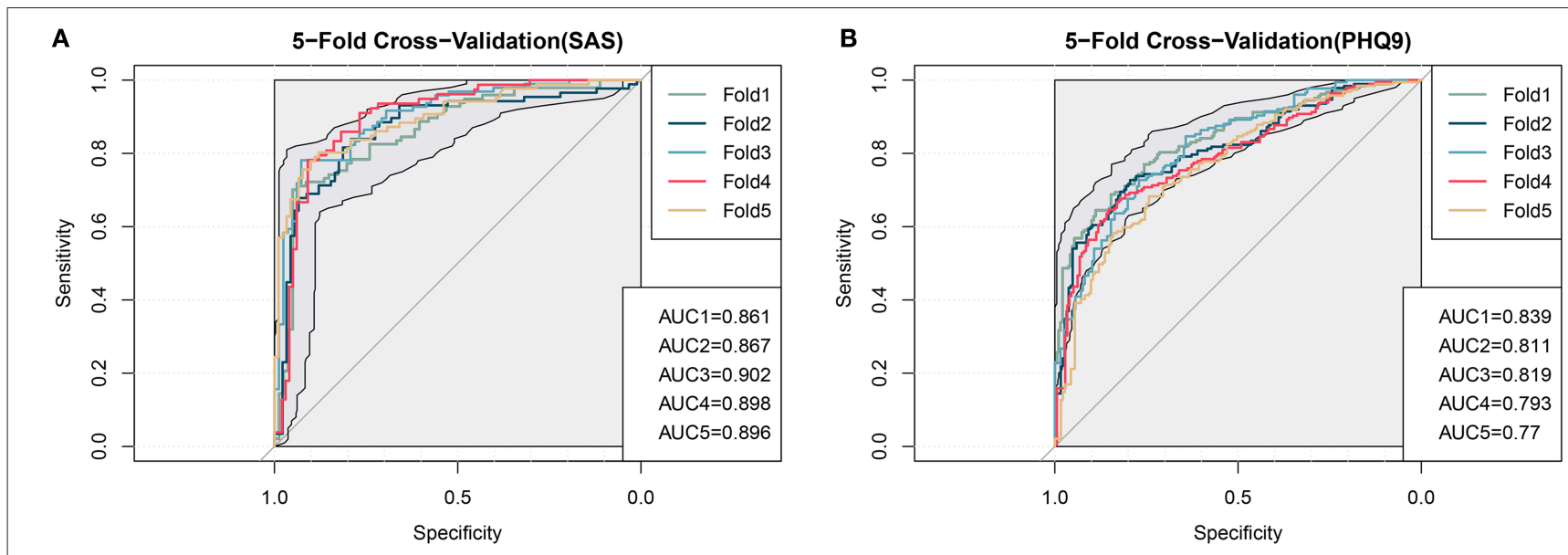

FIGURE 2 | Evaluation of the logistic model by 5-fold cross-validation. (A) anxiety; (B) depression. AUC, area under the curve.

anxiety and depression among college students. According to our findings, several preventive interventions should be mentioned. First, schools should provide professional psychological help for students suffering from COVID-19, especially having relatives or friends who have been infected. Schools should also provide more financial aids for students in poverty during COVID-19. Besides, schools should encourage students to develop healthy lifestyles, including daily exercise and lower alcohol use. Besides, schools should emphasize the importance of sleep, especially in this particular period. Finally, some strict regulations should be applied, such as wearing masks and taking temperature routinely. These measurements would even improve students' mental health. Simultaneously, schools should ensure that some services closely related to students' daily life, such as delivery service, will be maintained.

Previous studies have demonstrated that college students have been suffering extreme mental pressure during this pandemic, and proposed some countermeasures. Chi's study supported interventions promoting resilience, even remotely, to subjects with specific risk factors of developing poor mental health during COVID-19 or other pandemics with social isolation (Chi et al., 2020). Chen et al. found that isolation policy had a complex influence on the symptoms of obsessive-compulsive disorder, fear, hypochondria, depression, and neurasthenia via various 
factors and introduced a six-step intervention strategy to alleviate young people's psychological problems while in isolation (Chen et al., 2020). Similar studies were performed in many other countries, including the United States (Huckins et al., 2020; Son et al., 2020), Saudi Arabia (Alkhamees et al., 2020), India (Kapasia et al., 2020), Bangladesh (Khan et al., 2020), and Jordan (Naser et al., 2020). Huckins et al. reported that compared with U.S. college students in the prior academic terms, the Winter 2020 term individuals were more sedentary, anxious, and depressed. A wide variety of behaviors, including increased phone usage, decreased physical activity, and fewer locations visited, were associated with fluctuations in COVID-19 news reporting (Huckins et al., 2020).

Some previous studies have also reported some "hub influence factors" that serve as mediators between "ordinary influence factors" and psychological problems. For example, as mentioned above, Zhang et al. utilized $\mathrm{R}$ software's mediation package to find that the severity of the COVID-19 outbreak indirectly affects negative emotions by affecting sleep quality (Zhang et al., 2020). Such mediators also include resilience, social support, and coping (Yang et al., 2020a). These "hub factors" could be considered "targets" for psychological interventions, including psychological counsel to strengthen one's resilience and coping ability, social support from friends or family, and even medical intervention. For example, the appropriate application of some hypnotics has been proved to be effective for anxiety and depression patients without significant side effects (Yang et al., 2020b). The "sleep quality" factor derived in our study is one of the previously discovered "hub influence factors" (Zhang et al., 2020). Therefore, we highly suggested that schools encourage students to get enough sleep times and higher sleep quality.

In our study, we did not find any relationship between majors and the mental health of college students. However, many previous studies have reported that the psychological impact of the pandemic on college students majoring in psychology or medicine-related subjects is more significant. Guidotti et al. found that a notable percentage of neuropsychology trainees reported increased personal mental health symptoms (i.e., anxiety/depression; 74/54\%) as well as several other personal stressors (Guidotti Breting et al., 2020). Similar situations occurred in Nepal (Khanal et al., 2020; Shrestha, 2020) and China (Xiao et al., 2020). To conclude, as for psychological/medical students, COVID-19 might cause enormous psychological stress. And psychological interventions should be implemented. Besides, more clinical studies should be conducted to prove this point.

This study has some strengths. First, this is the first published article examining college students' mental status after school reopening to the best of our knowledge. Second, the subjects included in this study were from several different schools across China, which increases this study's universality. Third, the application of machine learning algorithms, including SMOTE, AIC, multivariate logistic regression, and ROC curves, is appropriate and reasonable, increasing the study's scientificity and reliability.

This study also has some limitations. First, the sample was relatively small. It is not easy to explore differences among schools. We just used the Chi-square test to prove no significant differences between city areas and rural areas. Second, some infrequent influence factors, which certain subjects mentioned, were not included in the questionnaire. Third, more psychological clinical trials should be performed to discover the susceptibility to other mental problems such as alexithymia (Tang et al., 2020,b) and PTSD after school reopening.

\section{CONCLUSION}

In this study, we performed a cross-sectional survey to investigate the prevalence of anxiety and depression among Chinese college students after school reopening and explored a series of factors influencing students' mental health. Many studies have demonstrated that college students have been suffering extreme mental pressure during the pandemic. For example, Cao et al. performed a cross-sectional study in China and found that $0.9 \%$ of the respondents were experiencing severe anxiety, $2.7 \%$ were experiencing moderate anxiety, and $21.3 \%$ were experiencing mild anxiety. Also, influence factors and some so-called "hub influence factors" were mentioned. Currently, the pandemic's control status varies worldwide. The situation of COVID-19 in China significantly improved and the school reopens. However, in some other countries, the school might reopen after a period of time. To the best of our knowledge, this is the first published article examining college students' mental status after school reopening. Therefore, we evaluated the school regulation measures based on college students' mental health, which could provide sensitive suggestions for school management worldwide.

\section{DATA AVAILABILITY STATEMENT}

The raw data supporting the conclusions of this article will be made available by the authors, without undue reservation.

\section{ETHICS STATEMENT}

The studies involving human participants were reviewed and approved by Ethics Committee of Shandong University. Written informed consent for participation was not required for this study in accordance with the national legislation and the institutional requirements.

\section{AUTHOR CONTRIBUTIONS}

Data collection was fulfilled by ZR, JG, and ZZ. Statistical analyses were performed by YX. The manuscript was written by ZR and YX. The study was designed by ZR, JG, and DL. The manuscript was revised by DL, $\mathrm{RH}$, and $\mathrm{CH}$. All authors read and approved the final manuscript.

\section{FUNDING}

This work was supported by the National Key Research and Development Program of China (No. 2017YFC0820203). 


\section{REFERENCES}

Alemany-Arrebola, I., Rojas-Ruiz, G., Granda-Vera, J., and Mingorance-Estrada, Á. C. (2020). Influence of COVID-19 on the perception of academic selfefficacy, state anxiety, and trait anxiety in college students. Front. Psychol. 11:570017. doi: 10.3389/fpsyg.2020.570017

Alkhamees, A. A., Alrashed, S. A., Alzunaydi, A. A., Almohimeed, A. S., and Aljohani, M. S. (2020). The psychological impact of COVID-19 pandemic on the general population of Saudi Arabia. Compr. Psychiatry 102:152192. doi: 10.1016/j.comppsych.2020.152192

Beijing News (2020). In Jiangxi province, all closed campus management has been abolished, combining "humanization" with scientific prevention and control. Available online at: https://www.bjnews.com.cn/detail/160042055315203.html (accessed November 25, 2020).

Cao, W., Fang, Z., Hou, G., Han, M., Xu, X., Dong, J., et al. (2020). The psychological impact of the COVID-19 epidemic on college students in China. Psychiatry Res. 287:112934. doi: 10.1016/j.psychres.2020.112934

Chen, B., Sun, J., and Feng, Y. (2020). How have COVID-19 isolation policies affected young people's mental health? - Evidence from Chinese college students. Front. Psychol. 11:1529. doi: 10.3389/fpsyg.2020.01529

Cheung, T., Wong, S. Y., Wong, K. Y., Law, L. Y., Ng, K., Tong, M. T., et al. (2016). Depression, anxiety and symptoms of stress among baccalaureate nursing students in hong kong: a cross-sectional study. Int. J. Environ. Res. Public Health 13:779. doi: 10.3390/ijerph13080779

Chi, X., Becker, B., Yu, Q., Willeit, P., Jiao, C., Huang, L., et al. (2020). Prevalence and psychosocial correlates of mental health outcomes among chinese college students during the Coronavirus disease (COVID-19) pandemic. Front. Psychiatry 11:803. doi: 10.3389/fpsyt.2020.00803

de Girolamo, G., Dagani, J., Purcell, R., Cocchi, A., and McGorry, P. D. (2012). Age of onset of mental disorders and use of mental health services: needs, opportunities and obstacles. Epidemiol. Psychiatr. Sci. 21, 47-57. doi: $10.1017 /$ S2045796011000746

Giannopoulou, I., Efstathiou, V., Triantafyllou, G., Korkoliakou, P., and Douzenis, A. (2020). Adding stress to the stressed: senior high school students' mental health amidst the COVID-19 nationwide lockdown in Greece. Psychiatry Res. 2020:113560. doi: 10.1016/j.psychres.2020.113560

Guan, W. J., Liang, W. H., Zhao, Y., Liang, H. R., Chen, Z. S., Li, Y. M., et al. (2020a). Comorbidity and its impact on 1590 patients with COVID-19 in China: a nationwide analysis. Eur. Respir. J. 55:2000547. doi: 10.1183/13993003.00547-2020

Guan, W. J., Ni, Z. Y., Hu, Y., Liang, W. H., Ou, C. Q., He, J. X., et al. (2020b). Clinical characteristics of Coronavirus disease 2019 in China. N. Engl. J. Med. 382, 1708-1720. doi: 10.1056/NEJMoa2002032

Guidotti Breting, L. M., Towns, S. J., Butts, A. M., Brett, B. L., Leaffer, E. B., and Whiteside, D. M. (2020). 2020 COVID-19 American Academy of Clinical Neuropsychology (AACN) Student Affairs Committee survey of neuropsychology trainees. Clin. Neuropsychol. 2020, 1-30. doi: 10.1080/13854046.2020.1809712

Huckins, J. F., daSilva, A. W., Wang, W., Hedlund, E., Rogers, C., Nepal, S. K., et al. (2020). Mental health and behavior of college students during the early phases of the COVID-19 pandemic: longitudinal smartphone and ecological momentary assessment study. J. Med. Internet Res. 22:e20185. doi: 10.31234/osf.io/4enzm

Kalok, A., Sharip, S., Abdul Hafizz, A. M., Zainuddin, Z. M., and Shafiee, M. N. (2020). The psychological impact of movement restriction during the COVID19 outbreak on clinical undergraduates: a cross-sectional study. Int. J. Environ. Res. Public Health 17:8522. doi: 10.3390/ijerph17228522

Kapasia, N., Paul, P., Roy, A., Saha, J., Zaveri, A., Mallick, R., et al. (2020). Impact of lockdown on learning status of undergraduate and postgraduate students during COVID-19 pandemic in West Bengal, India. Child. Youth Serv. Rev. 116:105194. doi: 10.1016/j.childyouth.2020.105194

Kecojevic, A., Basch, C. H., Sullivan, M., and Davi, N. K. (2020). The impact of the COVID-19 epidemic on mental health of undergraduate students in New Jersey, cross-sectional study. PLOS ONE 15:e0239696. doi: 10.1371/journal.pone.0239696

Kessler, R. C., Amminger, G. P., Aguilar-Gaxiola, S., Alonso, J., Lee, S., and Ustün, T. B. (2007). Age of onset of mental disorders: a review of recent literature. Curr. Opin. Psychiatry 20, 359-364. doi: 10.1097/YCO.0b013e32816ebc8c
Khan, A. H., Sultana, M. S., Hossain, S., Hasan, M. T., Ahmed, H. U., and Sikder, M. T. (2020). The impact of COVID-19 pandemic on mental health and wellbeing among home-quarantined Bangladeshi students: a cross-sectional pilot study. J. Affect. Disord. 277, 121-128. doi: 10.1016/j.jad.2020.07.135

Khanal, P., Devkota, N., Dahal, M., Paudel, K., and Joshi, D. (2020). Mental health impacts among health workers during COVID-19 in a low resource setting: a cross-sectional survey from Nepal. Global. Health 16:89. doi: 10.1186/s12992-020-00621-z

Kroenke, K., Spitzer, R. L., and Williams, J. B. (2001). The PHQ-9: validity of a brief depression severity measure. J. Gen. Intern. Med. 16, 606-613. doi: 10.1046/j.1525-1497.2001.016009606.x

Lattie, E. G., Adkins, E. C., Winquist, N., Stiles-Shields, C., Wafford, Q. E., and Graham, A. K. (2019). Digital mental health interventions for depression, anxiety, and enhancement of psychological well-being among college students: systematic review. J. Med. Internet Res. 21:e12869. doi: 10.2196/12869

Levin, M. E., Haeger, J. A., Pierce, B. G., and Twohig, M. P. (2017). Webbased acceptance and commitment therapy for mental health problems in college students: a randomized controlled trial. Behav. Modif. 41, 141-162. doi: $10.1177 / 0145445516659645$

Levin, M. E., Hayes, S. C., Pistorello, J., and Seeley, J. R. (2016). Web-based self-help for preventing mental health problems in universities: comparing acceptance and commitment training to mental health education. J. Clin. Psychol. 72, 207-225. doi: 10.1002/jclp.22254

Lungu, A., and Sun, M. (2016). Time for a change: college students' preference for technology-mediated versus face-to-face help for emotional distress. Telemed. J. E-Health 22, 991-1000. doi: 10.1089/tmj.2015.0214

Ma, Z., Zhao, J., Li, Y., Chen, D., Wang, T., Zhang, Z., et al. (2020). Mental health problems and correlates among 746217 college students during the coronavirus disease 2019 outbreak in China. Epidemiol. Psychiatr. Sci. 29:e181. doi: $10.1017 /$ S2045796020000931

Mazza, M. G., De Lorenzo, R., Conte, C., Poletti, S., Vai, B., Bollettini, I., et al. (2020). Anxiety and depression in COVID-19 survivors: role of inflammatory and clinical predictors. Brain Behav. Immun. 89, 594-600. doi: 10.1016/j.bbi.2020.07.037

Ministry of Education of the People's Republic of China (2020). 20 questions about the resumption of school and class under normalization of Epidemic prevention and controls (in Chinese). Available online at: http://www.moe.gov.cn/jyb_ xwfb/moe_1946/fj_2020/202006/t20200612_465535.html (accessed November $25,2020)$.

Naser, A. Y., Dahmash, E. Z., Al-Rousan, R., Alwafi, H., Alrawashdeh, H. M., Ghoul, I., et al. (2020). Mental health status of the general population, healthcare professionals, and university students during 2019 coronavirus disease outbreak in Jordan: a cross-sectional study. Brain Behav. 10:e01730. doi: 10.1002/brb3.1730

National Health Commission of the People's Republic of China (2020). Notification for the coronavirus disease (COVID-19) pandemic (in Chinese) Available online at: http://www.nhc.gov.cn/xcs/yqfkdt/gzbd_index.shtml (accessed November 22, 2020).

Oftedal, S., Kolt, G. S., Holliday, E. G., Stamatakis, E., Vandelanotte, C., Brown, W. J., et al. (2019). Associations of health-behavior patterns, mental health and self-rated health. Prev. Med. 118, 295-303. doi: 10.1016/j.ypmed.2018. 11.017

Pappa, S., Ntella, V., Giannakas, T., Giannakoulis, V. G., Papoutsi, E., and Katsaounou, P. (2020). Prevalence of depression, anxiety, and insomnia among healthcare workers during the COVID-19 pandemic: a systematic review and meta-analysis. Brain Behav. Immun. 88, 901-907. doi: 10.1016/j.bbi.2020.05.026

People's Daily (2020). To investigate and inspect the prevention before school reopening (in Chinese). Available online at: http://paper.people.com. $\mathrm{cn} / \mathrm{rmrb} / \mathrm{html} / 2020-06 / 05 / \mathrm{nw}$.D110000renmrb_20200605_3-02.htm (accessed November 25, 2020).

R Core Team (2020). R: A Language and Environment for Statistical Computing. Vienna: R Foundation for Statistical Computing. Available online at: https:// www.R-project.org/ (accessed September 26, 2020).

Seidel, E. J., Mohlman, J., Basch, C. H., Fera, J., Cosgrove, A., and Ethan, D. (2020). Communicating mental health support to college students during covid-19: an exploration of website messaging. J. Commun. Health 45, 1259-1262. doi: 10.1007/s10900-020-00905-w 
Shader, R. I. (2020). COVID-19 and depression. Clin. Ther. 42, 962-963. doi: 10.1016/j.clinthera.2020.04.010

Shrestha, S. L. (2020). Prevalence of psychological effect of COVID-19 on medical professionals in a tertiary care center. J. Nepal Med. Assoc. 58, 550-553. doi: 10.31729/jnma.5087

Son, C., Hegde, S., Smith, A., Wang, X., and Sasangohar, F. (2020). Effects of COVID-19 on college students mental health in the US: an interview-survey study. J. Med. Internet Res. 22:e21279. doi: 10.2196/21279

Tang, B., Xia, F., Tang, S., Bragazzi, N. L., Li, Q., Sun, X., et al. (2020). The effectiveness of quarantine and isolation determine the trend of the COVID19 epidemics in the final phase of the current outbreak in China. Int. J. Infect. Dis. 95, 288-293. doi: 10.1016/j.ijid.2020.03.018

Tang, W., Hu, T., Hu, B., Jin, C., Wang, G., Xie, C., et al. (2020a). Prevalence and correlates of PTSD and depressive symptoms one month after the outbreak of the COVID-19 epidemic in a sample of home-quarantined Chinese university students. J. Affect. Disord. 274, 1-7. doi: 10.1016/j.jad.2020.05.009

Tang, W., Hu, T., Yang, L., and Xu, J. (2020b). The role of alexithymia in the mental health problems of home-quarantined university students during the COVID-19 pandemic in China. Pers. Individ. Dif. 165:110131. doi: $10.1016 /$ j.paid. 2020.110131

Tu, H., Tu, S., Gao, S., Shao, A., and Sheng, J. (2020). Current epidemiological and clinical features of COVID-19; a global perspective from China. J. Infect. 81, 1-9. doi: 10.1016/j.jinf.2020.04.011

Velten, J., Bieda, A., Scholten, S., Wannemüller, A., and Margraf, J. (2018). Lifestyle choices and mental health: a longitudinal survey with German and Chinese students. BMC Public Health 18:632. doi: 10.1186/s12889-0185526-2

Vindegaard, N., and Benros, M. E. (2020). COVID-19 pandemic and mental health consequences: systematic review of the current evidence. Brain Behav. Immun. 89, 531-542. doi: 10.1016/j.bbi.2020.05.048

Walsh, R. (2011). Lifestyle and mental health. Am. Psychol. 66, 579-592. doi: $10.1037 /$ a0021769

Wang, M., Kou, C., Bai, W., Song, Y., Liu, X., Yu, W., et al. (2019). Prevalence and correlates of suicidal ideation among college students: a mental health survey in Jilin Province, China. J. Affect. Disord. 246, 166-173. doi: 10.1016/j.jad.2018.12.055

Webermann, A. R., and Murphy, C. M. (2020). How can psychology help reduce gender-based violence and misconduct on college campuses? Am. Psychol. doi: 10.1037/amp0000705. [Epub ahead of print].

World Health Organization (2020). Coronavirus disease (COVID-19) pandemic. Available online at: https://www.who.int/emergencies/diseases/novelcoronavirus-2019 (accessed November 22, 2020).

Xiao, H., Shu, W., Li, M., Li, Z., Tao, F., Wu, X., et al. (2020). Social distancing among medical students during the 2019 Coronavirus disease pandemic in China: disease awareness, anxiety disorder, depression, and behavioral activities. Int. J. Environ. Res. Public Health 17:5047. doi: 10.3390/ijerph17145047

Xin, M., Luo, S., She, R., Yu, Y., Li, L., Wang, S., et al. (2020). Negative cognitive and psychological correlates of mandatory quarantine during the initial COVID-19 outbreak in China. Am. Psychol. 75, 607-617. doi: 10.1037/amp0000692

Xingchen, H., Yingli, Z., and Wei, L. (2014). The reliability and validity test of the patient health questionnaire depression scale (PHQ-9) in adolescent (in Chinese). Psychol. J. Sichuan Province. 27, 357-360. Available online at: http://www.psychjm.net.cn/ch/reader/view_abstract.aspx?file_no= 201404021\&flag=1

Yang, D., Tu, C. C., and Dai, X. (2020a). The effect of the 2019 novel coronavirus pandemic on college students in Wuhan. Psychol. Trauma 12, 6-14. doi: 10.1037/tra0000930

Yang, L., Yu, Z., Xu, Y., Liu, W., Liu, L., and Mao, H. (2020b). Mental status of patients with chronic insomnia in China during COVID-19 epidemic. Int. J. Soc. Psychiatry 66, 821-826. doi: 10.1177/0020764020937716

Yongan, Z. (2012). Application of the Self-Rating Anxiety Scale in College Students in China (in Chinese). Master's thesis. Southern Medical University, Guangzhou, China.

Zhai, Y., and Du, X. (2020). Addressing collegiate mental health amid COVID-19 pandemic. Psychiatry Res. 288:113003. doi: 10.1016/j.psychres.2020.113003

Zhang, Y., Zhang, H., Ma, X., and Di, Q. (2020). Mental health problems during the COVID-19 pandemics and the mitigation effects of exercise: a longitudinal study of college students in China. Int. J. Environ. Res. Public Health 17:3722. doi: 10.3390/ijerph17103722

Zhou, S. J., Wang, L. L., Yang, R., Yang, X. J., Zhang, L. G., Guo, Z. C., et al. (2020). Sleep problems among Chinese adolescents and young adults during the coronavirus-2019 pandemic. Sleep Med. 74, 39-47. doi: 10.1016/j.sleep.2020.06.001

Zung, W. W. (1971). A rating instrument for anxiety disorders. Psychosomatics 12, 371-379. doi: 10.1016/S0033-3182(71)71479-0

Conflict of Interest: The authors declare that the research was conducted in the absence of any commercial or financial relationships that could be construed as a potential conflict of interest.

Copyright (C) 2021 Ren, Xin, Ge, Zhao, Liu, Ho and Ho. This is an open-access article distributed under the terms of the Creative Commons Attribution License (CC $B Y)$. The use, distribution or reproduction in other forums is permitted, provided the original author(s) and the copyright owner(s) are credited and that the original publication in this journal is cited, in accordance with accepted academic practice. No use, distribution or reproduction is permitted which does not comply with these terms. 